\title{
ESTABLECIENDO LAS BASES PARA PREVENIR EL CONSUMO TEMPRANO DE ALCOHOL: ANÁLISIS DE LAS ACTITUDES DE ADOLESCENTES DE 12 A 14 AÑOS
}

\author{
Carlos Teira \\ Universidade da Coruña \\ Alicia Risso \\ Universidade da Coruña \\ alicia.risso@udc.es
}

Recepción Artículo: 7 septiembre 2020 Admisión Evaluación: 15 noviembre 2020 Informe Evaluador 1: 25 noviembre 2020 Informe Evaluador 2: 23 noviembre 2020 Aprobación Publicación: 27 noviembre 2020

\section{RESUMEN}

En la actualidad, el consumo temprano de alcohol es una preocupación de salud pública por los problemas que puede ocasionar en los adolescentes, tanto a nivel físico, como social o psicológico. Por ello, la finalidad de este trabajo fue investigar sobre las actitudes subyacentes a los principales motivos de consumo de alcohol al comienzo de esa etapa evolutiva, ya que actúan como factores de riesgo de consumo prematuro. Para ello, se contó con una muestra de 181 adolescentes de ambos sexos residentes en la Comunidad Autónoma de Galicia, de entre 12 y 14 años de edad. La recogida de datos se realizó a partir de un cuestionario diseñado específicamente hoc para ser cubierto online, cuyas preguntas se agrupaban en tres dimensiones motivacionales: socialización, mejora y afrontamiento. El análisis de su consistencia interna mostró una elevada fiabilidad. Para el análisis de los resultados se hicieron contrastes de medias con pruebas post hoc y se estudiaron las correlaciones. Se observó que las variables que tienen una relación significativa con la evaluación afectiva de las expectativas 0 actitudes son la edad y la exposición previa al consumo y, en menor grado, el sexo. Además, se han podido detectar las dimensiones motivacionales y los ítems actitudinales concretos que pueden implicar un mayor riesgo de consumo futuro. Con esta información será posible desarrollar una propuesta metodológica de modelado de actitudes, que podría integrarse en programas de prevención destinados a evitar el consumo temprano de alcohol.

Palabras clave: prevención del uso de sustancias; adolescencia; actitudes de consumo de alcohol; comportamiento de consumo; educación sobre drogas

\section{ABSTRACT}

Establishing the basis for preventing early alcohol consumption: an analysis of the attitudes of adolescents from $\mathbf{1 2}$ to $\mathbf{1 4}$ years old. At present, early alcohol consumption is a public health concern 


\section{ESTABLECIENDO LAS BASES PARA PREVENIR EL CONSUMO TEMPRANO DE ALCOHOL: ANÁLISIS DE LAS ACTITUDES DE ADOLESCENTES DE 12 A 14 AÑOS}

because of the problems it can cause in adolescents, be it physical, social or psychological. Therefore, the purpose of this work was to investigate the attitudes underlying the main reasons for alcohol consumption at the beginning of that stage of development, since they act as risk factors for premature consumption. For this purpose, it was taken a sample of 181 adolescents of both sexes living in the Autonomous Community of Galicia, between 12 and 14 years old. The data was collected through a questionnaire specifically designed to be covered online, whose questions were grouped into three motivational dimensions: socialization, improvement and coping. The analysis of its internal consistency showed high reliability. For the analysis of the results, means were contrasted with post hoc tests and the correlations were studied. It was observed that the variables significantly related to the affective evaluation of expectations or attitudes are age and previous exposure to consumption and, to a lesser extent, sex. In addition, it has been possible to detect the motivational dimensions and specific attitudinal items that may imply a greater risk of future consumption. With this information it will be possible to develop a methodological proposal for attitude modeling, which could be integrated into prevention programs aimed at avoiding early alcohol consumption.

Keywords: substance use prevention; adolescence; alcohol drinking attitudes; drinking behaviour; drug education

La reducción del consumo de alcohol tiene una relevancia fundamental en la salud pública, dado que, como destaca la OMS en su último informe, su consumo abusivo causa alrededor de tres millones de muertes al año, el $5.3 \%$ del total mundial (World Health Organization, 2018). Asimismo, los estados de embriaguez correlacionan con problemas sociales como peleas, accidentes de tráfico, delincuencia, etc. (Bonomo et al., 2001; French y Maclean, 2006; García y Jiménez, 2018; Kuntsche, Plant, Plant, Miller y Gmel, 2008).

En España, la encuesta ESTUDES (Ministerio de Sanidad, Consumo y Bienestar Social, 2018), indica que el $77.9 \%$ de los adolescentes de entre 14 y 18 años ha consumido bebidas alcohólicas alguna vez en su vida y, como indica Lozano (2014), la edad media de comienzo está en torno a los 14 años. Este dato es muy relevante, dado que el inicio temprano del consumo de alcohol representa un importante factor de riesgo para el desarrollo de futuros patrones de consumo pernicioso. Estudios previos muestran que, en comparación con quienes inician su consumo a los 21 años, los que comienzan a beber a los 14 años 0 antes tienen, al menos tres veces más probabilidad de experimentar dependencia alcohólica en un futuro (Hingson, Heeren, Jamanka, y Howland, 2000; Hingson, Heeren y Winter, 2006).

Además, los adolescentes son más vulnerables al etanol que los adultos (Bonomo, Bowes, Coffey, Carlin y Patton, 2004), ya que se encuentran en un período crítico del desarrollo, en el cual el cerebro experimenta cambios estructurales y funcionales (Guerri y Pascual, 2010). Estudios al respecto sugieren que la exposición al alcohol durante la maduración cerebral actúa en perjuicio de la salud psicológica, social y física (Gmel, Rehm y Kuntsche, 2003), pudiendo acarrear deficiencias cerebrales y disfunciones cognitivas y comportamentales (Guerri y Pascual, 2010), afectando negativamente a la planificación, la capacidad viso-espacial y la memoria (Brown, Tapert, Granholm y Delis, 2000).

Puesto que el alcohol es una de las drogas preferidas en la adolescencia (Donovan, 2004; Ministerio de Sanidad, Consumo y Bienestar Social, 2018) y que en la mayoría de los casos el inicio del consumo se produce en esa etapa evolutiva (Kuntsche, Knibbe, Gmel y Engels, 2005), urge disponer de programas de prevención eficaces para evitar el consumo desde edades tempranas (Demant y Schierf, 2017; Larimer y Cronce, 2002), como expone la Estrategia Nacional sobre Adicciones 2017-2024 (Ministerio de Sanidad, Consumo y Bienestar Social, 2017).

Dichos programas deben partir del estudio de la etiología del consumo de alcohol (Kuntsche et al., 2005), tratando de determinar los factores que pueden desempeñar un papel crucial en la decisión inicial de probarlo (Copeland, Proctor, Terlecki, Kulesza y Williamson, 2014). Según McCambridge y Strang (2003), serán eficaces si se orientan a cambiar las percepciones y creencias asociadas al alcohol. De hecho, las investigaciones muestran que el estudio de las actitudes permite predecir las conductas de consumo (McPherson y Harris, 2013; 
Dormal, Maurage, Lannoy y D Hont, 2018), ya que pueden actuar como un factor de riesgo o de protección ante esas conductas (Beerli-Palacio, Martín-Santana, Díaz-Meneses, Fernández-Monroy y Galván-Sánchez, 2012), 10 cual suscita interés por intentar modelarlas en los programas preventivos.

En general, las actitudes hacia una conducta específica están condicionadas por factores como las creencias sobre las consecuencias que puedan tener (expectativas) y las evaluaciones afectivas (positivas y negativas) de las mismas (Ajzen, 2005). Según la teoría del aprendizaje social (Bandura, 1997) las personas pueden disponer de creencias y expectativas de las contingencias derivadas de una determinada conducta antes de haberla experimentado personalmente, desarrolladas tanto de forma directa como indirecta (Houben, Schoenmakers y Wiers, 2010).

Como indican las teorías del comportamiento planificado (Ajzen, 1991) y del valor de la expectativa (Hays, 1985), Ios adolescentes decidirán si llevar a cabo una conducta que todavía no han experimentado en función de las tendencias actitudinales o la valoración de las expectativas de que disponen en el momento. En este sentido, las actitudes implícitas y explícitas (Greenwald y Banaji, 1995; Nosek y Smyth, 2007), contribuyen de forma independiente a los comportamientos relacionados con el alcohol (Rooke, Hine y Thorsteinsson, 2008), lo que explicaría que, aunque los bebedores sean conscientes de las consecuencias negativas que entraña el consumo y manifiesten una volición real de abstenerse, acaben bebiendo (Geng y Quian, 2011). Con el consumo reiterado de la substancia, las asociaciones automáticas se vuelven más fuertes y pueden abocar a conductas adictivas (Tiffany, 1990; Wiers et al., 2007), lo cual sugiere la importancia de mediar en las actitudes lo antes posible, para evitar estas asociaciones automatizadas.

Diferentes variables parecen afectar en las actitudes de los adolescentes. Así, el aumento de la edad muestra una correlación directa con el incremento de las expectativas positivas, e inversa respecto a las expectativas negativas (Copeland et al., 2014; Dunn y Goldman, 2000), siendo las positivas factores de riesgo más determinantes para predecir el inicio temprano del consumo de alcohol (Pilatti, Godoy y Brussino, 2011). Otras variables mediadoras son el hecho de consumir alcohol y la cantidad ingerida (Lac y Donaldson, 2016), y también varios estudios indican que los varones muestran unas expectativas positivas más fuertes hacia la bebida que las mujeres (Véase Patrick, Wray-Lake, Finlay y Maggs, 2010).

Como expone el modelo motivacional, la decisión de beber es una combinación de procesos emocionales y racionales, que viene motivada por el cambio afectivo que se espera lograr llevando a cabo dicha conducta (Kuntsche et al., 2005). Investigaciones previas sobre los motivos de consumo identifican tres tendencias motivacionales de riesgo que abocan a los adolescentes a beber alcohol: a) Ios motivos de mejora (beber para mejorar habilidades 0 activarse), que están relacionados con el consumo excesivo de alcohol; b) los motivos de afrontamiento (beber para animarse cuando se está de mal humor y para olvidar los problemas), que están asociados con consumo abusivo y dependencia; y c) los motivos sociales (beber para ser más sociable y celebrar ocasiones especiales en compañía), que son los más comunes en el inicio de consumo (Kuntsche et al., 2005; Lac y Donaldson, 2016).

Tanto las actitudes hacia el alcohol (Beerli-Palacio et al., 2012) como los motivos de iniciación de consumo son aspectos fundamentales para la prevención (Kuntsche y Müller, 2012), teniendo en cuenta la influencia que tienen en el comportamiento y lo maleables que son cuando todavía no se ha generado dependencia. Por ello, para crear un programa de prevención eficaz, se debe comenzar por determinar qué aspectos actitudinales y motivacionales concretos representan factores de riesgo de consumo (Lac y Donaldson, 2016), para intervenir en consecuencia y tratar de revertirlos en factores de protección (National Institute on Drug Abuse (2003). Como la prevención radicaría en el cambio actitudinal hacia el alcohol, podría emplearse un modelo de doble proceso como el Condicionamiento Evaluativo, puesto que: opera en los dos procesos de cambio actitudinal (implícitos y deliberativos o proposicionales), es resistente a la extinción, ha demostrado su efectividad para reducir conductas de consumo, y puede emplearse para revertir la valencia afectiva de un estímulo, a modo de contracondicionamiento (De houwer, Thomas y Baeyens, 2001, Houben, Schoenmakers y Wiers, 2010; Hu, Gawronski y Balas, 2017; Tello, Bocage-Barthélémy, Dandaba, Jaafari y Chatard, 2018; Zerhouni, Bègue, Comiran y Wiers, 2018). 


\section{ESTABLECIENDO LAS BASES PARA PREVENIR EL CONSUMO TEMPRANO DE ALCOHOL: ANÁLISIS DE LAS ACTITUDES DE ADOLESCENTES DE 12 A 14 AÑOS}

En base a lo hasta aquí expuesto, los objetivos de esta investigación fueron, por un lado, analizar las variables que inciden en las actitudes subyacentes a los principales motivos de consumo de alcohol de los adolescentes y, por otro, inferir las dimensiones motivacionales e ítems actitudinales que implican un mayor riesgo de ingesta alcohólica. En el futuro, continuando con esta línea de investigación y con la información y los datos obtenidos aquí, se intentará esbozar un modelo de prevención del consumo basado en técnicas de condicionamiento evaluador.

\section{MÉTODO}

Para esta investigación se empleó una metodología de encuesta de tipo muestral, explicativa y transversal.

\section{Participantes}

La población objetivo de esta investigación eran los y las adolescentes de los primeros cursos de ES0 (1214 años), residentes en la Comunidad Autónoma de Galicia. Participaron voluntariamente 187 estudiantes de centros públicos (56 \%), concertados (43\%) y privados (1\%) de las cuatro provincias gallegas. De entre todas las respuestas, seis no fueron sido válidas, con lo que la muestra fue de $N=181$. De estos, 122 eran mujeres (67 \%) y $59(33 \%)$ varones. El $56 \%(n=102)$ tenían 14 años, el $28 \%(n=50) 13$ y un $16 \%(n=29) 12$, siendo la media de edad 13.4 años $(D T=.75)$. No se encontraron diferencias en la distribución de estas variables ni por provincias ni por tipo de centro, ni tampoco entre los datos muestrales y los poblacionales.

\section{Instrumento}

Para la recogida de datos se empeló una encuesta confeccionada ad hoc que constaba de tres partes. En la primera se explicaban las condiciones del estudio y la garantía de anonimato para los participantes; después había preguntas de carácter demográfico (edad, sexo, municipio de residencia, curso y tipo de centro en el que estudiaban) y otras relativas a si habían consumido alcohol alguna vez y en qué situación, así como la cantidad y el motivo por el que suelen beber. La tercera parte incluía un cuestionario de 31 ítems con escala de respuesta tipo Likert ( 1 = Muy en desacuerdo y 5 = Muy de acuerdo) para conocer las actitudes de los adolescentes hacia el consumo de alcohol. Su estructura contemplaba las tres dimensiones que representan los principales motivos de consumo de riesgo (Kuntsche et al., 2005): Socialización, Mejora y Afrontamiento. La mayor parte de los ítems fueron de elaboración propia. En otros, el contenido se basó en cuestionarios existentes (Camacho et al., 2013; Pilatti, Godoy y Brussino, 2012), si bien se reformularon y adaptaron a los criterios de redacción que se habían establecido: formular todas las afirmaciones bajo un enfoque positivo (puesto que se pretende analizar las actitudes más positivas que puedan conllevar al consumo) y en tercera persona (encuestando a los sujetos sobre lo que creen que ocurre a otras personas cuando beben, para evitar dar por hecho que todos los adolescentes han consumido). En la Tabla 1 pueden verse todos los ítems y el número de orden en el que aprecían. El formato de presentación fue a través de Google Forms, para ser cubierto online, de forma autoadmistrada. 
Tabla 1. Ítems del cuestionario Actitudes Hacia el Consumo de Alcohol (AHCA)

\begin{tabular}{|c|c|}
\hline Orden & Texto \\
\hline \multicolumn{2}{|c|}{ Socialización (Soc) } \\
\hline 1 & La gente que bebe alcohol es más aceptada por los demás \\
\hline 4 & $\begin{array}{l}\text { La gente cuando bebe alcohol es más "extrovertida" (las personas } \\
\text { extrovertidas son las que hablan más y dicen lo que piensan y sienten al resto } \\
\text { de la gente con mayor facilidad) }\end{array}$ \\
\hline 8 & La gente que bebe alcohol tiene más poder en su grupo de amigos \\
\hline 10 & El alcohol ayuda a la gente a hacer más amigos \\
\hline 14 & La gente que toma alcohol es más agradable \\
\hline 15 & Cuando las personas beben alcohol se vuelven más cariñosas con los demás \\
\hline 19 & Las personas que beben se sienten más cómodas para hacer nuevos amigos \\
\hline 23 & La gente que bebe liga más \\
\hline 26 & La gente cuando bebe siente que tiene más amigos \\
\hline 27 & Mucha gente bebe porque también lo hacen sus amigos \\
\hline 29 & Es bueno que la gente beba alcohol para brindar con sus amigos o su familia \\
\hline \multicolumn{2}{|r|}{ Mejora (Mej) } \\
\hline 2 & Las personas que beben alcohol se divierten más en las fiestas \\
\hline 5 & La gente que bebe alcohol es más feliz \\
\hline 7 & Las personas que beben alcohol tienen mejores ideas \\
\hline 11 & Las personas cuando beben alcohol sienten cambios agradables \\
\hline 12 & Cuando la gente toma alcohol está más atenta a lo que sucede a su alrededor \\
\hline 13 & Las personas que beben son más fuertes \\
\hline 18 & La gente bebe por primera vez para averiguar que se siente \\
\hline 20 & La gente cuando bebe se vuelve más atractiva \\
\hline 21 & La gente que bebe tiene una mejor salud \\
\hline 22 & Las personas que beben son más maduras \\
\hline 30 & La gente que bebe alcohol sabe más de la vida \\
\hline \multicolumn{2}{|r|}{ Afrontamiento (Afr) } \\
\hline 3 & Tomar alcohol ayuda a que la gente olvide sus sentimientos negativos \\
\hline 6 & Cuando las personas beben alcohol sienten menos estrés \\
\hline 9 & $\begin{array}{l}\text { Cuando las personas beben alcohol no se sienten avergonzadas por las cosas } \\
\text { que hacen }\end{array}$ \\
\hline 16 & El alcohol hace que la gente se tranquilice \\
\hline 17 & El alcohol distrae a la gente de sus problemas \\
\hline 24 & Cuando la gente bebe aguanta más los insultos de los demás \\
\hline 25 & $\begin{array}{l}\text { El alcohol hace que las personas se atrevan a hacer cosas que no harían si no } \\
\text { bebiesen }\end{array}$ \\
\hline 28 & El alcohol ayuda a las personas a superar sus miedos \\
\hline 31 & $\begin{array}{l}\text { Las personas beben para transformar sus pensamientos negativos en otros } \\
\text { más positivos }\end{array}$ \\
\hline
\end{tabular}

\section{Procedimiento}

Los datos se recogieron durante ocho semanas, a través de internet. Para obtener la muestra se contactó con más de 180 IES de toda la Galicia, informado de los objetivos de la investigación y enviando un enlace a la encuesta para que los docentes de los cursos correspondientes a las edades de la investigación lo compartiesen 


\section{ESTABLECIENDO LAS BASES PARA PREVENIR EL CONSUMO TEMPRANO DE ALCOHOL: ANÁLISIS DE LAS ACTITUDES DE ADOLESCENTES DE 12 A 14 AÑOS}

con su alumnado. Para facilitar el acceso de los estudiantes, también se confeccionó un Twitter exclusivo con un enlace directo y se animó a la distribución a fin de obtener un efecto "bola de nieve", así aunque la colaboración institucional fue escasa, se contó con una muestra bastante amplia y con características similares a las de la población objetivo.

\section{Análisis de datos}

Los datos fueron extraídos en una hoja de cálculo, en la cual se realizó la primera aproximación al análisis, eliminando las encuestas no válidas (únicamente un 3\%), la categorización de las respuestas a las preguntas abiertas y la incorporación de nuevas variables derivadas de conjuntos de preguntas. Por ejemplo, de "¿Has consumido alcohol alguna vez?" y "¿Cuantas copas sueles beber normalmente cuando sales de fiesta?", se creó la variable "exposición al consumo", que divide al total de participantes en tres grupos: nunca probaron el alcohol, únicamente lo han probado y consumen con frecuencia. Otra fue la variable referente a la cantidad de consumo habitual, mediante la cual se operativizan los resultados de la pregunta "¿Cuantas copas sueles beber normalmente cuando sales de fiesta?" en base a la Unidad de Bebida Estándar (UBE), de modo que se estima la cantidad de gramos de alcohol puro ingeridos. También se computó la puntuación total y las puntuaciones parciales en las tres dimensiones de motivos de consumo que abarca el cuestionario. Los análisis estadísticos descriptivos e inferenciales se realizaron con el IBM SPSS 26 (licencia de campus).

\section{RESULTADOS}

En primer lugar, hay que señalar que el cuestionario AHCA, obtuvo una buena fiabilidad, tanto para el conjunto de los ítems (Alfa de Cronbach=.91), como para las dimensiones que los agrupaban: Socialización (Soc)= .83, Mejora (Mej) $=.79$ y Afrontamiento $($ Afr $)=.80$.

Por otro lado, los resultados muestran que un alto porcentaje de los adolescentes de entre 12 y 14 años ha probado el alcohol alguna vez (61\%), y que más de la quinta parte (22 \%) consume con frecuencia.

Entre quienes suelen consumir, la preferencia es clara hacia las bebidas de alta graduación, como ron, ginebra y vodka (80 \%) ante otras como el vino y la cerveza (20\%), y cabe destacar que un $27.5 \%$ acostumbra beber dos copas y un $30 \%$ cuatro o más. Respecto a los motivos de consumo reportados explícitamente, el $65.5 \%$ lo hace por motivos de mejora, el 31 \% socialización y sólo el 3.5 \% por afrontamiento.

Cabe destacar que las puntuaciones medias más altas y sin relación con otras variables fueron las de los ítems 17 (3.4), 25 (4.08) y 27 (4.21), mientras que las más bajas fueron las del 7 (1.34), 12 (1.36), 13 (1.34), 21 (1.15.

En cuanto a la importancia de las variables objeto de investigación, en la variable Sexo, no hubo diferencias entre varones y mujeres ni en la puntuación total ni en las dimensiones, ni en casi ningún ítem. Únicamente se observaron diferencias significativas en tres: el relativo a que la gente que bebe sabe más de la vida, en el que la valoración media de los varones fue mayor (ítem 30: $M_{V}=1.51, M_{M}=1.24, t(179)=2.37, p=.019, d=.34$ ), y Ios que hacían referencia a que el alcohol ayuda a hacer más amigos/as (ítem 10: $M_{V}=2.02, M_{M}=2.53, t(179)$ $=-0.52, p=.013, d=.24$ ) y que la gente que bebe es más agradable (ítem 14: $M_{V}=1.51, M_{M}=1.79, t(179)=$ $-1.78, p=.049, d=.30$ ), en los que las adolescentes dieron valoraciones más altas. En todos los casos el tamaño del efecto ( $d$ de Cohen) fue pequeño, por lo que se podría concluir que las diferencias de actitud hacia el consumo de alcohol en adolescentes de 12-14 años son mínimas.

La edad, en cambio, sí mostró tener relevancia. Por un lado, correlaciona significativamente $(p<.01)$ con la puntuación total $(r=.26)$ y con los motivos de Socialización ( $r=.33)$ y Mejora $(r=.22)$. Así, a medida que aumenta la edad, se incrementa la puntuación total $\left(M_{12}=67.3, M_{13}=68.3\right.$ y $\left.M_{14}=77.6 ; F(2,179)=7.17, p<.001\right)$ y las de las dimensiones Socialización $\left(M_{12}=24.62, M_{13}=26.46\right.$ y $\left.M_{14}=31.01 ; F(2,178)=10.69, p<.001\right)$ y Mejora $\left(M_{12}=17.48, M_{13}=18.60\right.$ y $\left.M_{14}=20.07 ; F(2,178)=3.87, p<.023\right)$. Además, las pruebas post hoc mostraron diferencias estadísticamente significativas para los ítems y entre las edades que muestra la Tabla 2. Las 
diferencias observadas fueron entre el grupo de 14 y los otros dos, no existiendo entre los 12 y los 13 años. El tamaño del efecto fue grande en todos los casos $\left(p^{2}>.14\right)$ y la potencia observada $=1.00$.

Tabla 2. Resultados significativos $(p<.05)$ de las comparaciones múltiples post hoc (DMS) del ANOVA para grupos de la variable Edad

\begin{tabular}{|c|c|c|c|c|c|c|c|c|c|}
\hline Ítems & Edad I & Edad J & $\begin{array}{c}D M(\mathrm{I}- \\
\mathrm{J})\end{array}$ & $\mathrm{EE}$ & Ítems & Edad I & Edad $\mathbf{J}$ & $\begin{array}{c}D M(\mathrm{I}- \\
\mathrm{J})\end{array}$ & \\
\hline 1. (Soc) & 14 & 12 & .63 & .27 & \multirow{2}{*}{ 18. (Mej) } & 14 & 12 & .63 & .26 \\
\hline \multirow{2}{*}{ 10. (Soc) } & 14 & 12 & 1.10 & .26 & & 14 & 13 & .53 & .21 \\
\hline & 14 & 13 & 0.86 & .21 & \multirow{2}{*}{ 19. (Soc) } & 14 & 12 & .66 & .27 \\
\hline \multirow{2}{*}{ 11. (Mej) } & 14 & 12 & 0.58 & .22 & & 14 & 13 & .48 & .22 \\
\hline & 14 & 13 & 0.52 & .18 & 22. (Mej) & 14 & 12 & .29 & .13 \\
\hline \multirow{2}{*}{ 14. (Soc) } & 14 & 12 & 0.69 & .69 & \multirow{2}{*}{ 23. (Soc) } & 14 & 12 & .64 & .27 \\
\hline & 14 & 13 & 0.45 & .45 & & 14 & 13 & .47 & .22 \\
\hline \multirow{2}{*}{ 15. (Soc) } & 14 & 12 & 0.77 & .77 & 29. (Soc) & 14 & 12 & .92 & .26 \\
\hline & 14 & 13 & 0.56 & .56 & \multirow{2}{*}{ 31. (Afr) } & 14 & 12 & .51 & .26 \\
\hline 16. (Afr) & 14 & 12 & 0.47 & .21 & & 14 & 13 & .64 & .21 \\
\hline
\end{tabular}

Nota: $D M=$ Diferencia de medias, $E E=$ Error estándar

Por lo que se refiere a las actitudes relacionadas con la exposición previa al consumo de alcohol se observaron diferencias importantes entre quienes nunca han probado el alcohol (G1), quienes lo han probado pero no consumen (G2) y el grupo de consumidores frecuentes. El análisis de varianza y las pruebas post hoc mostraron que en la puntuación total existen diferencias entre las medias de los tres grupos $\left(M_{\mathrm{G} 1}=68.32, M_{\mathrm{G} 2}=75.68 \mathrm{y}\right.$ $\left.M_{\mathrm{G} 3}=81.17 ; F(2,179)=17.53, p<.001\right)$. Examinando las dimensiones, puede verse que en Socialización el $\mathrm{G} 1$ se diferencia de los otros dos, mientras que en Mejora es el G3 el que se distingue de los demás; en Afrontamiento solo hay diferencias entre el G1 y el G3. Un resumen de estos resultados, así como los relativos a los ítems que arrojaron diferencias estadísticamente significativas en relación con la variable Exposición al consumo de alcohol, puede verse en la Tabla 3. El grupo de quienes consumen habitualmente tuvo promedios más altos (indicativos de actitudes más positivas hacia el consumo de alcohol) que los que no consumen y éstos más que los que no han probado bebidas alcohólicas. 
ESTABLECIENDO LAS BASES PARA PREVENIR EL CONSUMO TEMPRANO DE ALCOHOL:

ANÁLISIS DE LAS ACTITUDES DE ADOLESCENTES DE 12 A 14 AÑOS

Tabla 3. Resultados significativos $(p<.05)$ de las comparaciones múltiples post hoc (DMS) del ANOVA para los grupos "nunca han probado el alcohol" (G1), "han probado pero no consumen" (G2) y consumidores frecuentes (G3).

\begin{tabular}{|c|c|c|c|c|c|c|c|c|c|}
\hline & I & $\mathrm{J}$ & $D M(\mathrm{I}-\mathrm{J})$ & $E E$ & & I & $\mathrm{J}$ & $D M(\mathrm{I}-\mathrm{J})$ & $E E$ \\
\hline \multirow{2}{*}{ Socialización } & 3 & 1 & 0.69 & 0.13 & \multirow{2}{*}{ Mejora } & 3 & 2 & 0.41 & 0.11 \\
\hline & 2 & 1 & 0.45 & 0.14 & & 3 & 1 & 0.48 & 0.09 \\
\hline Afrontamiento & 3 & 1 & 0.49 & 0.14 & \multirow{3}{*}{14 (Soc) } & & & & \\
\hline \multirow[b]{2}{*}{ 2. (Mej) } & 3 & 2 & 0.85 & 0.31 & & 3 & 1 & 0.75 & 0.18 \\
\hline & 3 & 1 & 1.09 & 0.24 & & 2 & 1 & 0.45 & 0.19 \\
\hline \multirow{2}{*}{ 3. (Afr) } & 3 & 1 & 0.85 & 0.25 & \multirow{2}{*}{ 15. (Soc) } & 3 & 1 & 1.09 & 0.22 \\
\hline & 2 & 1 & 0.79 & 0.27 & & 2 & 1 & 0.57 & 0.24 \\
\hline \multirow{2}{*}{ 4. (Soc) } & 3 & 1 & 0.77 & 0.22 & \multirow{2}{*}{ 19. (Soc) } & 3 & 2 & 0.70 & 0.30 \\
\hline & 2 & 1 & 0.52 & 0.24 & & 3 & 1 & 0.98 & 0.23 \\
\hline \multirow{2}{*}{ 5. (Mej) } & 3 & 2 & 0.87 & 0.22 & \multirow{2}{*}{ 20. (Mej) } & 3 & 2 & 0.50 & 0.21 \\
\hline & 3 & 1 & 1.01 & 0.17 & & 3 & 1 & 0.50 & 0.16 \\
\hline \multirow{2}{*}{ 6. (Afr) } & 3 & 2 & 0.79 & 0.30 & \multirow{2}{*}{ 23. (Soc) } & 3 & 1 & 1.02 & 0.23 \\
\hline & 3 & 1 & 1.19 & 0.23 & & 2 & 1 & 0.54 & 0.25 \\
\hline \multirow{3}{*}{ 10. (Soc) } & 3 & 2 & 0.78 & 0.29 & \multirow{3}{*}{ 29. (Soc) } & 3 & 1 & 0.91 & 0.23 \\
\hline & 3 & 1 & 1.36 & 0.22 & & 2 & 1 & 0.65 & 0.25 \\
\hline & 2 & 1 & 0.59 & 0.25 & & & & & \\
\hline \multirow{2}{*}{ 11. (Mej) } & 3 & 2 & 0.82 & 0.24 & \multirow{2}{*}{ 30. (Mej) } & 3 & 2 & 0.34 & 0.17 \\
\hline & 3 & 1 & 0.83 & 0.19 & & 3 & 1 & 0.36 & 0.13 \\
\hline
\end{tabular}

Finalmente, cabe mencionar que las respuestas a los ítems relativos a Tener más poder en el grupo, Sentir que se tienen más amigos/as, Tener mejores ideas, Estar más atentos, Ser más fuertes, Tener mejor salud, No tener vergüenza de lo que se hace, Aguantar los insultos, y Superar miedos, fueron similares; independientemente de la edad, el sexo, y de consumir o no alcohol.

\section{DISCUSIÓN}

En el presente estudio se ha observado que más de la mitad de los adolescentes de entre 12 y 14 años ya ha probado el alcohol en alguna ocasión (60.9\%), una cifra que se aproxima a la ofrecida por la encuesta ESTUDES (Ministerio de Sanidad, Consumo y Bienestar Social, 2018) realizada a jóvenes de 14 a 18 años (77.9 \%). Este dato es bastante negativo teniendo en cuenta los riesgos que implica el consumo a edades tan tempranas del desarrollo, tanto de cara a padecer una futura dependencia al alcohol (Hingson et al., 2000; Hingson et al., 2006) como para el desarrollo cerebral y el bienestar (Guerri y Pascual, 2010; Gmel et al., 2003; Brown et al., 2000). También se pudo confirmar que muchos adolescentes han consumido de forma excesiva (Kuntsche et al., 2005) pues los participantes que afirmaron consumir alcohol mostraron una media elevada: 4.7 UBE, equivalente a 47 gramos de alcohol (cantidad estipulada como consumo de riesgo en varones y perjudicial en mujeres, si este fuese mantenido en el tiempo como patrón de ingesta diaria: Organización Panamericana de la Salud, 2008).

Teniendo en cuenta lo anterior y lo perniciosa que puede resultar la ingesta etílica en una etapa crítica y vulnerable como la adolescencia (Bonomo et al., 2004; Guerri y Pascual, 2010), se hace patente la necesidad de investigar sobre las conductas de consumo de cara a diseñar futuras actividades de prevención realmente efectivas para evitar el consumo prematuro de alcohol (Larimer y Cronce, 2002; Demant y Schierf, 2017; Kuntsche y Müller, 2012; Lac y Donaldson, 2016), como propone la Estrategia Nacional sobre Adicciones 2017-2024 (Ministerio de Sanidad, Consumo y Bienestar Social, 2017). 
Investigaciones anteriores resaltan la importancia que tienen las actitudes en las conductas de consumo de alcohol (Ajzen, 2005; Dormal et al., 2018; McPherson y Harris, 2013), puesto que la decisión de ingerirlo es mediada por la valoración de las expectativas de las que se dispone en el momento (Ajzen, 1991; Hays, 1985), y porque la conducta en sí está motivada por el cambio afectivo que se espera lograr Ilevandola a cabo (Kuntsche et al., 2005). Por ello, los objetivos de este estudio eran analizar las variables que inciden en las actitudes subyacentes a los principales motivos de consumo de alcohol de los adolescentes e inferir las dimensiones motivacionales e ítems actitudinales que implican un mayor riesgo de ingesta alcohólica.

Respecto a la variable sexo, las diferencias halladas se dieron en únicamente tres ítems, mostrando las mujeres actitudes más positivas en dos de ellos, lo que no permite respaldar los resultados de investigaciones que afirman que la valoración afectiva de las expectativas es más fuerte en los varones (Patrick et al., 2010).

En cuanto a la variable edad, se confirmó su relación con actitudes positivas hacia el consumo, al igual que ocurría en anteriores estudios (Copeland et al., 2014; Pilatti et al., 2011). Así, el análisis de correlaciones mostró que esas actitudes aumentaban significativamente a medida que se incrementaba la edad, tanto en la puntuación total, como en las dimensiones de socialización y mejora. Además se identificaron diferencias significativas entre los grupos de edad en 12 items, de entre los cuales siete pertenecían a la dimensión de socialización, tres a la de mejora y dos a la de afrontamiento. Esto sugiere que la dimensión de socialización es la que experimenta un mayor incremento actitudinal con el crecimiento, por lo que debería ser atendida en prevención lo antes posible.

En cuanto al consumo, se confirma la influencia de la variable de exposición al consumo en cambio actitudinal (Lac y Donaldson, 2016). De entre los resultados, destaca la importancia que tiene el simple hecho de probar el alcohol, pues se hallaron diferencias significativas entre el grupo que no había probado nunca y el que sí Io había hecho, tanto en la puntuación total, como en la dimensión de socialización y en siete ítems, lo cual denota la necesidad de prevenir, ya no solo el consumo frecuente de alcohol, sino también el hecho de probarlo. Esta primera exposición, aunque sea aislada, al alcohol aumenta su imagen positiva, lo cual facilitaría la decisión conductual de consumo futuro. Además, seis de los ítems que mostraron diferencias significativas pertenecían a la dimensión de socialización, lo cual resalta la necesidad de intervenir en ella.

Los resultados también mostraron que, una vez el consumo se hace frecuente las actitudes se vuelven aún más positivas, pues se encontraron diferencias significativas entre los bebedores frecuentes con respecto a los que sólo han probado el alcohol; tanto en puntuación total, como en la dimensión de mejora y en ocho de los ítems. De entre estos, cabe destacar que cinco eran de la dimensión mejora. De ello podemos inferir que, para prevenir que los adolescentes que sólo han probado el alcohol acaben consumiendo de forma frecuente y desarrollen unas tendencias actitudinales automatizadas (Tiffany, 1990; Wiers et al., 2007), se debería trabajar principalmente el cambio actitudinal en esta dimensión.

\section{CONCLUSIONES}

Estos resultados nos permiten esbozar unas directrices para el diseño de futuros programas preventivos para adolescentes, constatando que se deberían trabajar los contenidos actitudinales en función de las características concretas que tenga el grupo al que se dirija. Teniendo en cuenta que el consumo de alcohol se inicia en torno a Ios 14 años (ESTUDES, 2018; Lozano, 2014) y que las actitudes positivas se incrementan con la edad y la exposición al consumo, lo idóneo sería enfocar los programas de prevención a adolescentes que todavía no hayan probado (12 013 años). De este modo, se debería anteponer el modelado de las actitudes que más se incrementan con la edad, así como las que aumentan a raíz del primer consumo, priorizando los contenidos concretos de los ítems de la dimensión de socialización que coinciden en ambas variables. Como metodología para el programa, podría emplearse un modelo de doble proceso de cambio actitudinal como el Condicionamiento Evaluativo (Zerhouni et al., 2018) puesto que ya ha demostrado su eficacia para reducir las conductas de consumo (Houben et al., 2010; Tello et al., 2018).

En síntesis, esta investigación ha permitido discernir las dimensiones motivacionales e ítems actitudinales que pueden suponer factores de riesgo de consumo (Beerli-Palacio et al., 2012; Lac y Donaldson, 2016) y que 


\section{ESTABLECIENDO LAS BASES PARA PREVENIR EL CONSUMO TEMPRANO DE ALCOHOL: ANÁLISIS DE LAS ACTITUDES DE ADOLESCENTES DE 12 A 14 AÑOS}

deberían ser trabajados por futuros programas de prevención, con el objetivo de revertir su efecto, consiguiendo convertirlas en factores de protección como expone el manual de principios para la prevención del NIDA (2003), en pos de evitar el inicio de consumo de alcohol de los adolescentes y velar por su bienestar social, físico y psicológico (Gmel, Rehm y Kuntsche, 2003).

\section{REFERENCIAS BIBLIOGRÁFICAS}

Ajzen, I. (2005). Attitudes, personality and behavior. New York: Open University Press.

Ajzen I. (1991). The theory of planned behavior. Organizational Behavior and Human Decision Processes. Academic Press, 50(2), 179-211. https://doi.org/10.1016/0749-5978(91)90020-T

Bandura, A. (1977). Social Learning Theory. Englewood Cliffs, NJ: Prentice-Hall.

Beerli-Palacio, A., Martín-Santana, J., Díaz-Meneses, G., Fernández-Monroy, M., \& Galván-Sánchez, I. (2012). A model of attitudes, beliefs, emotions and values to explain the spanish youth street binge drinking phenomena. International Review on Public and Nonprofit Marketing, 9(2), 181-197. https://doi.org/10.1007/s12208012-0088-y

Bonomo, Y. A., Bowes, G., Coffey, C., Carlin, J. B., \& Patton, G. C. (2004). Teenage drinking and the onset of alcohol dependence: A cohort study over seven years. Addiction, 99(12), 1520-1528. https://doi.org/10.1111/j.1360-0443.2004.00846.x

Bonomo, Y., Coffey, C., Wolfe, R., Lynskey, M., Bowes, G., \& Patton, G. (2001). Adverse outcomes of alcohol use in adolescents. Addiction, 96(10), 1485-1496. https://doi.org/10.1046/j.1360-0443.2001.9610148512.x

Brown, S. A., Tapert, S. F., Granholm, E., \& Delis, D. C. (2000). Neurocognitive functioning of adolescents: Effects of protracted alcohol use. Alcoholism, Clinical and Experimental Research, 24(2), 164-171. https://doi.org/10.1097/00000374-200002000-00005

Camacho, L., Mezquita, L., Ibáñez, M. I., Moya, J., Villa, H., Viruela, A. M., \& Ortet, G. (2013). Spanish adaptation of the expectancy questionnaire (EQ) about alcohol effects in adolescents. Psicothema, 25(4), 529-535. https://doi.org/10.7334/psicothema2012.354

Copeland, A. L., Proctor, S. L., Terlecki, M. A., Kulesza, M., \& Williamson, D. A. (2014). Do positive alcohol expectancies have a critical developmental period in pre-adolescents? Journal of Studies on Alcohol and Drugs, 75(6), 945-952. https://doi.org/10.15288/jsad.2014.75.945

De Houwer, J., Thomas, S., \& Baeyens, F. (2001). Associative learning of likes and dislikes: A review of 25 years of research on human evaluative conditioning. Psychological Bulletin, 127(6), 853-869. https://doi.org/10.1037/0033-2909.127.6.853

Demant, J., \& Schierff, L. M. (2017). Five typologies of alcohol and drug prevention programmes. A qualitative review of the content of alcohol and drug prevention programmes targeting adolescents. Drugs: Education, Prevention and Policy, 26(1), 32-39. https://doi.org/10.1080/09687637.2017.1347147

Donovan, J. E. (2004). Adolescent alcohol initiation: A review of psychosocial risk factors. Journal of Adolescent Health, 35(6), 7-18. https://doi.org/10.1016/S1054-139X(04)00066-7

Dormal, V., Maurage, P., Lannoy, S., \& D’Hondt, F. (2018). Positive attitude toward alcohol predicts actual consumption in young adults: An ecological implicit association test. Journal of Studies on Alcohol and Drugs, 79(5), 733-740. https://doi.org/10.15288/jsad.2018.79.733

French, M. T., \& Maclean, J. C. (2006). Underage alcohol use, delinquency, and criminal activity. Health Economics, 15(12), 1261-1281. https://doi.org/10.1002/hec.1126

García, N. D. \& Jiménez, M.V. (2018). Consumo de alcohol, conducta antisocial e impulsividad en adolescentes españoles. Acta Colombiana de Psicología, 21(2), 121-130. https://doi.org/10.14718/ACP.2018.21.2.6

Geng, L., \& Qian, B. (2011). Implicit and explicit cognition of chinese heroin abusers. Social Behavior and Personality: An International Journal, 39(4), 433-443. https://doi.org/10.2224/sbp.2011.39.4.433 
Guerri, C., \& Pascual, M. (2010). Mechanisms involved in the neurotoxic, cognitive, and neurobehavioral effects of alcohol consumption during adolescence. Alcohol, 44(1), 15-26. https://doi.org/10.1016/j.alcohol.2009.10.003

Gmel, G., Rehm, J., \& Kuntsche, E. (2003). Binge drinking in Europe: definitions, epidemiology, and consequences. Sucht, 49(2), 105-116. https://psycnet.apa.org/doi/10.1024/suc.2003.49.2.105

Greenwald, A. G., \& Banaji, M. R. (1995). Implicit social cognition: Attitudes, self-esteem, and stereotypes. Psychological Review, 102(1), 4-27. https://doi.org/10.1037/0033-295X.102.1.4

Hays, R. (1985). An integrated Value Expectancy theory of alcohol and other drug use. British Journal of Addiction, 80(4), 379-384. https://doi.org/doi:10.1111/j.1360-0443.1985.tb03009.x

Hingson, R. W., Heeren, T., Jamanka, A., \& Howland, J. (2000). Age of drinking onset and unintentional injury involvement after drinking. Jama, 284(12), 1527-1533. https://doi.org/10.1001/jama.284.12.1527

Hingson, R. W., Heeren, T., \& Winter, M. R. (2006). Age at drinking onset and alcohol dependence: Age at onset, duration, and severity. Archives of Pediatrics \& Adolescent Medicine, 160(7), 739-746. https://doi.org/10.1001/archpedi.160.7.739

Houben, K., Schoenmakers, T. M., \& Wiers, R. W. (2010). I didn't feel like drinking but I don't know why: The effects of evaluative conditioning on alcohol-related attitudes, craving and behavior. Addictive Behaviors, 35(12), 1161-1163. https://doi.org/10.1016/j.addbeh.2010.08.012

Hu, X., Gawronski, B., \& Balas, R. (2017). Propositional versus dual-process accounts of evaluative conditioning: II. The effectiveness of counter-conditioning and counter-instructions in changing implicit and explicit evaluations. Social Psychological and Personality Science, 8(8), 858-866. https://doi.org/10.1177/1948550617691094

Kuntsche, E., Knibbe, R., Gmel, G., \& Engels, R. (2005). Why do young people drink? A review of drinking motives. Clinical Psychology Review, 25(7), 841-861. https://doi.org/10.1016/j.cpr.2005.06.002

Kuntsche, E., \& Müller, S. (2012). Why do young people start drinking? motives for first-time alcohol consumption and links to risky drinking in early adolescence. European Addiction Research, 18(1), 34-39. https://doi.org/10.1159/000333036

Kuntsche, S., Plant, M. L., Plant, M. A., Miller, P., \& Gmel, G. (2008). Spreading or concentrating drinking occasions - who is most at risk? European Addiction Research, 14(2), 71-81. https://doi.org/10.1159/000113721

Lac, A., \& Donaldson, C. D. (2016). Alcohol attitudes, motives, norms, and personality traits longitudinally classify nondrinkers, moderate drinkers, and binge drinkers using discriminant function analysis. Addictive behaviors, 61, 91-98. https://doi.org/10.1016/j.addbeh.2016.05.006

Larimer, M. E., \& Cronce, J. M. (2002). Identification, prevention and treatment: A review of individual-focused strategies to reduce problematic alcohol consumption by college students. Journal of Studies on Alcohol. Supplement, (14), 148-163. https://doi.org/10.15288/jsas.2002.s14.148

Lozano Galván, E. (2014). Consumo de tabaco y alcohol en jóvenes estudiantes de ciclo formativo. International Journal of Developmental and Educational Psychology, 1(1), 215 -228. https://doi.org/10.17060/ijodaep.2014.n1.v1.365

McCambridge, J., \& Strang, J. (2004). The efficacy of single session motivational interviewing in reducing drug consumption and perceptions of drug related risk and harm among young people: Results from a multi site cluster randomized trial. Addiction, 99(1), 39-52. https://doi.org/10.1111/j.1360-0443.2004.00564.x

McPherson, A., \& Harris, L. M. (2013). Implicit and explicit attitudes to alcohol in alcohol dependent and nonalcohol dependent samples. Journal of Psychopathology \& Behavioral Assessment. 35, 389-393. https://doi.org/10.1007/s10862-013-9345-6

Ministerio de Sanidad, Consumo y Bienestar Social. (2017). Estrategia Nacional sobre Adicciones 2017-2024 (680-18-086-4). Disponible en: https://pnsd.sanidad.gob.es/pnsd/estrategiaNacional/home.htm 
Ministerio de Sanidad, Consumo y Bienestar Social. (2018). Encuesta sobre Uso de Drogas en Enseñanzas Secundarias en España (ESTUDES) 1994-2018. Disponible en: https://pnsd.sanidad.gob.es/profesionales/sistemasInformacion/sistemalnformacion/encuestas_ESTUDES.htm

National Institute on Drug Abuse. (2003). Preventing Drug Use among Children and Asolescents. (No. 044212[A]). Disponible en: https://www.drugabuse.gov/sites/default/files/preventingdruguse_2.pdf

Nosek, B. A., \& Smyth, F. L. (2007). A multitrait-multimethod validation of the implicit association test. Experimental Psychology, 54(1), 14-29. https://doi.org/10.1027/1618-3169.54.1.14

Organización Panamericana de la Salud (2008). Alcohol y atención primaria de la salud. Washington. DC: OPS. Disponible en: https://www.who.int/substance_abuse/publications/alcohol_atencion_primaria.pdf

Patrick, M. E., Wray-Lake, L., Finlay, A. K., \& Maggs, J. L. (2010). The long arm of expectancies: Adolescent alcohol expectancies predict adult alcohol use. Alcohol \& Alcoholism, 45(1), 17-24. https://doi.org/10.1093/alcalc/agp066

Pilatti, A., Godoy, J., \& Brussino, S. (2011). Expectativas hacia el alcohol y consumo de alcohol en niños y adolescentes de Argentina. International Journal of Psychology and Psychological Therapy, 11(1), 13-32. https://www.ijpsy.com/volumen11/num1/280.html

Pilatti, A., Godoy, J. C., \& Brussino, S. A. (2012). Análisis factorial confirmatorio del cuestionario de expectativas hacia el alcohol para adolescentes (CEA-A). Acta Colombiana de Psicología, 15(2), 11-20. https://actacoIombianapsicologia.ucatolica.edu.co/article/view/262

Rooke, S. E. (2008). Examining adolescent and adult substance use from a dual process cognitive perspective. (Doctoral Thesis). University of New England, Biddeford. https://rune.une.edu.au/web/handle/1959.11/4062

Tello, N., Bocage-Barthélémy, Y., Dandaba, M., Jaafari, N. \& Chatard, A. (2018). Evaluative conditioning: A brief computer-delivered intervention to reduce college student drinking. Addictive Behaviors, 82, 14-18 https://doi.org/10.1016/j.addbeh.2018.02.018

Tiffany, S. T. (1990). A cognitive model of drug urges and drug-use behavior. Psychological Review, 97(2), 147168. https://doi.org/10.1037/0033-295X.97.2.147

Wiers, R. W., Bartholow, B. D., van den Wildenberg, E., Thush, C., Engels, R. C., Sher, K. J., . . Stacy, A. W. (2007). Automatic and controlled processes and the development of addictive behaviors in adolescents: A review and a model. Pharmacology, Biochemistry and Behavior, 86(2), 263-283. https://doi.org/10.1016/j.pbb.2006.09.021

World Health Organization (2018). Global status report on alcohol and health 2018: executive summary. World Health Organization. https://apps.who.int/iris/handle/10665/312318

Zerhouni, 0., Bègue, L., Comiran, F., \& Wiers, R. W. (2018). Controlled and implicit processes in evaluative conditioning on implicit and explicit attitudes toward alcohol and intentions to drink. Addictive Behaviors, 76, 335-342. https://doi.org/10.1016/j.addbeh.2017.08.026 